\title{
MANGROVE SPECIES AND STAND MAPPING IN GAZI BAY (KENYA) USING QUICKBIRD SATELLITE IMAGERY
}

\author{
Neukermans Griet ${ }^{1,2}$, Farid Dahdouh-Guebas' ${ }^{1}$, James Kairo ${ }^{3}$ and Nico Koedam² \\ 1 Biocomplexity Research Focus c/o \\ 2 Vrije Universiteit Brussel, Laboratory of Plant Biology and Nature Management, Mangrove \\ Management Group, Pleinlaan 2, B-1050 Brussel, Belgium \\ E-mail: gneukerm@vub.ac.be \\ ${ }^{3}$ Kenya Marine and Fisheries Research Institute, PO Box 81651, Mombasa, Kenya
}

Stand recognition (delineation and labelling) and species mapping are cornerstones of forest inventory mapping and key elements to forest management decision making. We present an automated method for mangrove stand recognition and species mapping based on fuzzy per-pixel classification techniques.

Mapping of the present distribution of mangrove species in Gazi Bay (Kenya) was done using supervised Maximum Likelihood fuzzy classification of a QuickBird satellite image. Species recordings were obtained during a field mission in July-August 2003 with the Point-Centred-Quarter-Method (PCQM). The overall accuracy if the species map is $72 \%$, where the two socio-economically most important species are mapped with user accuracies above $85 \%$. Mangrove stand maps are obtained through supervised fuzzy classification of the multispectral satellite image, convolution of an appropriate window size and subsequent elimination of patches covering less than 0.05ha. The automated stand boundaries were compared to visual delineations done by an expert interpreter. The quality of the correspondence between visual and automated stand boundaries was assessed based on the quantity of overlap one has with the other. The correspondence varied from perfect, over good, to poor matches. An overall correspondence of $64 \%$ was obtained for visual labelling of stands versus automated labelling (classification) based on dominant species and total cover. When only dominant species were taken into account, the overall accuracy of stand labelling increased to $86 \%$. Automated stand delineation and labelling are of a quality suitable for operational use in mangrove forest management. 